\title{
Effect of magnetic field on nanofluid free convection in Conical Partially Annular Space
}

\author{
Abderrahmane AISSA ${ }^{1}$, Mohamed Amine MEDEBBER ${ }^{1}$, Khaled Al-Farhany ${ }^{2}$, Mohammed SAHNOUN ${ }^{1}$, Ali $\mathrm{Khaleel}$ \\ Kareem $^{3}$ Mohammed EL GANAOUI ${ }^{4}$, \\ 1 Laboratoire de Physique Quantique de la Matière et Modélisation Mathématique (LPQ3M), Université Mustapha Stambouli de \\ Mascara, Algeria \\ 2 Department of mechanical engineering, University of Al-Qadisiyah, Al-Qadisiyah, Iraq \\ 3 Department of Mechanical engineering, University of Najaf, Iraq \\ 4 Laboratoire énergétique de Longwy (FJV/LERMAB), Université de Lorraine, France
}

\begin{abstract}
Natural convection of a magneto hydrodynamic nanofluid in a porous cavity in the presence of a magnetic field is investigated. The two vertical side walls are held isothermally at temperatures $\mathrm{Th}$ and Tc, while the horizontal walls of the outer cone are adiabatic. The governing equations obtained with the Boussinesq approximation are solved using Comsol Multiphysics finite element analysis and simulation software. Impact of Rayleigh number (Ra), Hartmann number (Ha) and nanofluid volume fraction $(\varnothing)$ are depicted. Results indicated that temperature gradient increases considerably with enhance of Ra and $\varnothing$ but it reduces with increases of $\mathrm{Ha}$.
\end{abstract}

\section{Introduction}

The problems of natural convection of modern fluids filled with nano-particles in different cavities have been the subject of many studies. This is due to the important role it plays in many technical and engineering applications such as, solar energy collectors, nuclear engineering, cooling of electrical components, geothermal energy extraction, food industries and etc. the problem of the low thermal conductivity of the working fluid such as air and water used in thermal equipments has been solved by adding metal nanoparticles.

Many researchers focused their investigation on the heat transfer and fluid flow behaviour in a rectangular cavity. One of the well documented studies the natural convection in rectangular enclosures differentially heated filled with air was presented by Behnia et al. [1] numerically studied the combined radiation and natural convection in a rectangular cavity with a transparent wall and containing a nonparticipating fluid. They showed that the radiation tends to accelerate the flow and this tendency increases with the increase of the Rayleigh number. Subsequently, Balaji et Venkateshan [2] analysed the same problem for a square cavity with opaque external surfaces. The results obtained show that the surface radiations effect the convective transfer. Although other studies concerning this type of coupling are carried out in different geometrical configurations and for various boundary conditions [3-6], practically they all converge on the same conclusions made previously concerning the effect of surface radiation on the thermal, dynamic fields and heat transfer.

Actually, many studies have been focused on the problems of natural convection in different cavities filled with nanofluid. Khanafer et al. [7] investigated analytical model to find out the natural convection heat transfer in nanofluid. Sheikha M. Al-Weheibi et al [8] analysed unsteady natural convection flow and heat transfer inside a trapezoidal enclosure filled with nine different types of nanofluid having various shapes of the nanoparticle. They founded that Heat transfer rate increases with the increase of the aspect ratio of the trapezoidal enclosure and with increased volume fraction of the nanoparticles in the base fluid.

Based on the above-mentioned works, and published results according to our best knowledge that there is no works has been published yet, devoted to studying features of free convection heat transfer in such conical geometry $(\mathrm{X}=\mathrm{h} / \mathrm{H}=0.5)$ : the inner cylinder height is different than the outer cone. Therefore, we focus our attention to study the laminar free convection heat transfer in a cylindrical cavity partially annular filled with $\mathrm{Cu}$-water nanofluid under radial magnetic field. The aim here was to investigate effects of pertinent parameters such as Rayleigh number $(103 \leq \mathrm{Ra} \leq 106)$, Hartmann number $(0 \leq \mathrm{Ha} \leq 100)$, and solid volume fraction is fixed $\phi=0.04$ on the flow and the heat transfer in the conical cavity

\section{Physical \& mathematical formulation}

\footnotetext{
* Corresponding author: aissa86@gmail.com
} 
In this present problem the geometry is schematized for studied the flow produced by natural convection in a vertical conical partial annulus; where the annulus is filled with air. The analysis domain is delimited by two concentric cone and a cylinder with isothermal walls of the inner and outer axial height $\mathrm{h}$ and $\mathrm{H}$, respectively.. The top and bottom walls of the outer cone are considered adiabatic, as shown in Fig. 1.

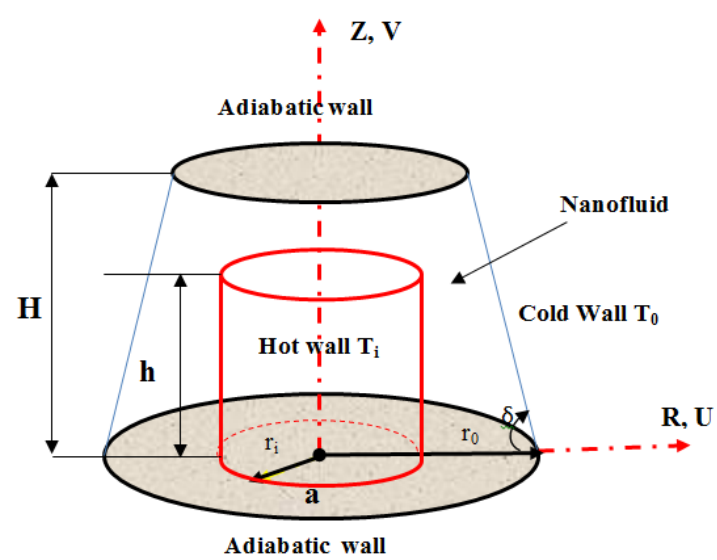

Fig. 1: Physical model of the problem

\subsection{Governing Equations:}

The equations governing the problem are written in dimensional form as follows:

The continuity equation for the two directions of the magnetic field:

$$
\frac{\partial(r u)}{\partial r}+\frac{\partial(r v)}{\partial z}=0
$$

When the magnetic field is applied radially:

Equation of motion according to $\mathrm{r}$ :

$$
\begin{aligned}
& u \frac{\partial u}{\partial r}+v \frac{\partial u}{\partial z}=\frac{1}{\rho_{n f}}\left[-\frac{\partial p}{\partial r}+\mu_{n f}\left(\frac{1}{r} \frac{\partial}{\partial r}\left(r \frac{\partial u}{\partial r}\right)+\right.\right. \\
& \left.\left.\frac{\partial^{2} u}{\partial z^{2}}-\sigma_{n f} B_{0}^{2} u\right)\right]
\end{aligned}
$$

Equation of motion according to z:

$$
\begin{aligned}
& u \frac{\partial v}{\partial r}+v \frac{\partial v}{\partial z}=\frac{1}{\rho_{n f}}\left[-\frac{\partial P}{\partial z}+\mu_{n f}\left(\frac{1}{r} \frac{\partial}{\partial r}\left(r \frac{\partial v}{\partial r}\right)+\right.\right. \\
& \left.\left.+\frac{\partial^{2} v}{\partial z^{2}}+(\rho \beta)_{n f} g\left(T-T_{c}\right)\right)\right]
\end{aligned}
$$

When the magnetic field is applied axially:

Equation of motion according to $\mathrm{r}$ :

$$
\begin{aligned}
& u \frac{\partial u}{\partial r}+v \frac{\partial u}{\partial z}= \\
& \frac{1}{\rho_{n f}}\left[-\frac{\partial p}{\partial r}+\mu_{n f}\left(\frac{1}{r} \frac{\partial}{\partial r}\left(r \frac{\partial u}{\partial r}\right)+\frac{\partial^{2} u}{\partial z^{2}}\right)\right]
\end{aligned}
$$

Equation of motion according to $\mathrm{z}$ :

$$
\begin{aligned}
& u \frac{\partial v}{\partial r}+v \frac{\partial v}{\partial z}=\frac{1}{\rho_{n f}}\left[-\frac{\partial p}{\partial z}+\mu_{n f}\left(\frac{1}{r} \frac{\partial}{\partial r}\left(r \frac{\partial v}{\partial r}\right)+\right.\right. \\
& \left.\left.\frac{\partial^{2} v}{\partial z^{2}}+(\rho \beta)_{n f} g\left(T-T_{c}\right)-\sigma_{n f} B_{0}^{2} v\right)\right]
\end{aligned}
$$

Energy equation in both directions of the magnetic field:

$u \frac{\partial T}{\partial r}+v \frac{\partial T}{\partial z}=\alpha_{n f}\left[\frac{1}{r} \frac{\partial}{\partial r}\left(r \frac{\partial T}{\partial r}\right)+\frac{\partial^{2} T}{\partial z^{2}}\right]$

The Thermophysical properties of nanofluid are determined by the correlations of Khanafer et al. [7]. The physical properties of the pure fluid (water) and those of the nanoparticles $(\mathrm{Cu})$ are given in Table 1

Table 1. Thermophysical properties of nanofluid

\begin{tabular}{|c|c|c|}
\hline & Water pure & Cuivre $(\mathrm{Cu})$ \\
\hline $\mathrm{Pr}$ & 6.2 & \\
\hline$\rho\left(\mathrm{kg} \cdot \mathrm{m}^{-3}\right)$ & 997.1 & 8933 \\
\hline $\mathrm{C}_{\mathrm{p}}\left(\mathrm{J} \cdot \mathrm{kg}^{-1} \cdot \mathrm{K}^{-1}\right)$ & 4179 & 385 \\
\hline $\mathrm{k}\left(\mathrm{W} \cdot \mathrm{m}^{-1} \cdot \mathrm{K}^{-1}\right)$ & 0.613 & 401 \\
\hline$\beta\left(\mathrm{K}^{-1}\right)$ & $21 \times 10^{-5}$ & $1.67 \times 10^{-5}$ \\
\hline
\end{tabular}

The thermal diffusivity of equation (6) is given by:

$$
\alpha_{n f}=k_{n f} /\left(\rho C_{p}\right)_{n f}
$$

The electrical conductivity of the nanofluid is given by:

$$
\sigma_{n f}=(1-\emptyset) \sigma_{f}+\emptyset \sigma_{p}
$$

The effective density of the nanofluid is expressed by:

$$
\rho_{n f}=(1-\emptyset) \rho_{f}+\emptyset \rho_{p}
$$

The heat capacity of the nanofluid is expressed by:

$$
\left(\rho C_{p}\right)_{n f}=(1-\emptyset)\left(\rho C_{p}\right)_{f}+\emptyset\left(\rho C_{p}\right)_{p}
$$

The coefficient of thermal expansion of the nanofluid is given by:

$$
(\rho \beta)_{n f}=(1-\emptyset)(\rho \beta)_{f}+\emptyset(\rho \beta)_{p}
$$

The dynamic viscosity of nanofluid is modeled by Brinkman [9].

$$
\begin{gathered}
\mu_{n f}=\mu_{f}(1-\emptyset)^{-2.5} \\
k_{n f}=k_{f}\left[\frac{\left(k_{\text {eff }}+2 k_{f}\right)-2 \emptyset\left(k_{f}-k_{\text {eff }}\right)+(1+\delta)^{\mathrm{a}}}{\left(k_{\text {eff }}+2 k_{f}\right)+\emptyset\left(k_{f}-k_{\text {eff }}\right)+(1+\delta)^{3}}\right]
\end{gathered}
$$

By introducing dimensionless parameters defined by:

$$
\begin{aligned}
& R=\frac{r}{R_{0}}, \quad Z=\frac{z}{R_{0}}, \quad U=\frac{w}{\left(\alpha_{f} / R_{0}\right)}, \\
& V=\frac{v}{\left(\kappa_{f} / R_{0}\right)},
\end{aligned}
$$




$$
\begin{gathered}
P=\frac{\bar{p} R_{0}^{3}}{\rho_{n f} \varpi_{f}^{3}}, \theta=\frac{T-T_{c}}{T_{h}-T_{r}}, R a=\frac{g \beta_{f} R_{0}^{g}\left(T_{h}-T_{c}\right)}{v_{f} \alpha_{f}}, \\
H a=B_{0} R_{0} \sqrt{\frac{\sigma_{n f}}{\rho_{n f} v_{f}}}, \quad \operatorname{Pr}=\frac{v_{f}}{\alpha_{f}} \quad \text { (17) }
\end{gathered}
$$

The dimensionless form of the governing equations (1) (6) is presented by:

$$
\frac{\partial(U \varphi)}{\partial R}+\frac{\partial(V \varphi)}{\partial Z}=\frac{\partial}{\partial R}\left(\Gamma_{\varphi} \frac{\partial \varphi}{\partial R}\right)+\frac{\partial}{\partial Z}\left(\Gamma_{\varphi} \frac{\partial \varphi}{\partial z}\right)+
$$

Where $\varphi$ replaces the dimensionless parameters $\mathrm{U}, \mathrm{V}$ and $\theta$ while $\Gamma \varphi$ et $S \varphi$ are respectively the terms diffusion and source. These variables are given in Table 2 .

The governing equations are accompanied with the following boundary conditions:

Axis of symmetry:

$$
\text { A } R=0 \quad \mathrm{U}=\frac{\partial \mathrm{V}}{\partial \mathrm{R}}=0 \frac{\partial \theta}{\partial \mathrm{R}}=0
$$

Adiabatic side wall:

$$
\text { A } \mathrm{R}=1 \quad \mathrm{U}=\mathrm{V}=0 \quad \frac{\partial \theta}{\partial \mathrm{R}}=0
$$

Hot bottom wall:

$$
\text { A } \mathrm{Z}=0 \quad \mathrm{U}=\mathrm{V}=0 \quad \theta=1
$$

Cold upper wall:

$$
\mathrm{A} Z=\frac{\mathrm{H}}{\mathrm{R}_{\mathrm{0}}} \mathrm{U}=\mathrm{V}=0 \quad \theta=0
$$

\subsection{Validation}

In order to validate the present results, the numerical results must be validated with the results published in the open literature. Validation with the published paper by Sajjadi et al. [10] has been done. The results are compared with MWCNT- $-\mathrm{Fe}_{3} \mathrm{O}_{4}$ /water hybrid, MHD natural convection in a porous medium and geometry presented by Sajjadi et al. The results are illustrated in Table 2.

Table 2. A comparison of the average Nusselt number with previous work for $\varphi=0, \mathrm{Ha}=0$

\begin{tabular}{|c|c|c|}
\hline \multicolumn{3}{|c|}{ Ha=0 } \\
\hline Ra & Present & Sajjadi et al. [22] \\
\hline $10^{3}$ & 1.01 & 1.1 \\
\hline $10^{4}$ & 2.11 & 2.03 \\
\hline $10^{5}$ & 3.6 & 3.66 \\
\hline
\end{tabular}

\section{Results and discussion}

In this section, the results of numerical simulations are presented with an objective to understand the effect of
Hartmann number on streamlines, isotherms, the dimensionless y-velocity and average Nusselt number. The Rayleigh number (Ra), Hartmann number (Ha), and the solid volume fraction $(\phi)$ are assumed to be in the following ranges: $103 \leq \mathrm{Ra} \leq 106, \quad 0 \leq \mathrm{Ha} \leq 120$, and $0 \leq \phi \leq 0.06$.

\subsection{Effect of Hartmann Number on the Streamlines and Isothermes}

The enclosure is filled with water-Cu nanofluid with a solid volume fraction of $\phi=0.04$. Figures 2 , 3, show, respectively, the streamlines and isotherms for five Rayleigh numbers $(\mathrm{Ra}=103,104,105$, and 106) and Hartmann numbers ( $\mathrm{Ha}=0,25,50$ and 100$)$.
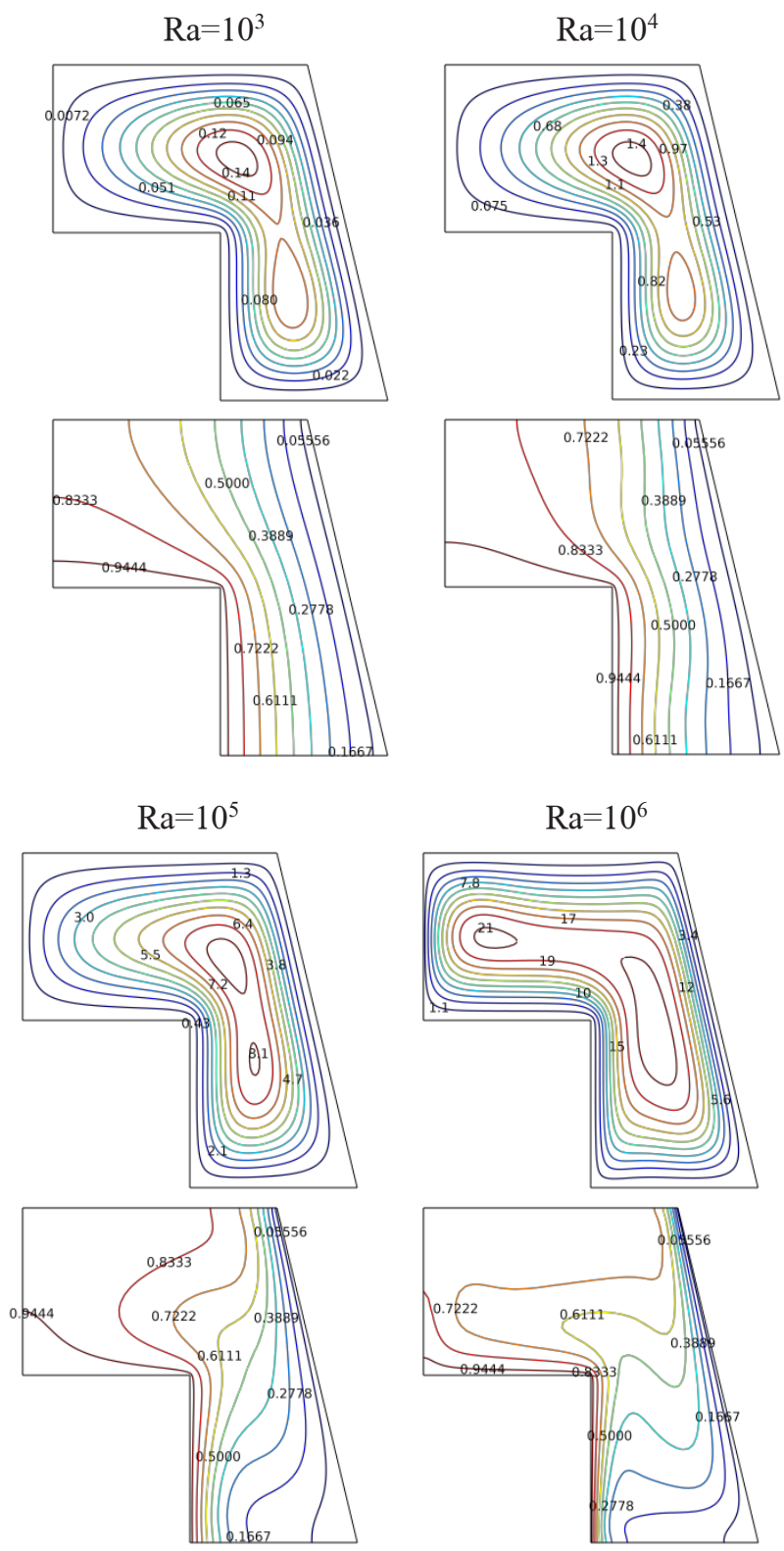

Fig. 2: Isotherm and streamlines for different $\mathrm{Ra}$ at $\mathrm{Ha}=0, \varphi=0.04$ 
For different Hartmann numbers and low values of Rayleigh number, we observe (fig 2) that the conduction regime is predominant with vertical isotherms and weak clockwise circulation in the cavity. The Circulation intensity is weaker due to the weak buoyancy forces.
$\mathrm{Ha}=50$
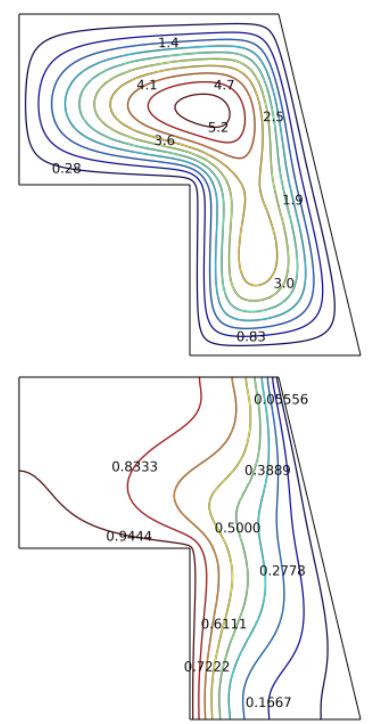

$\mathrm{Ha}=100$

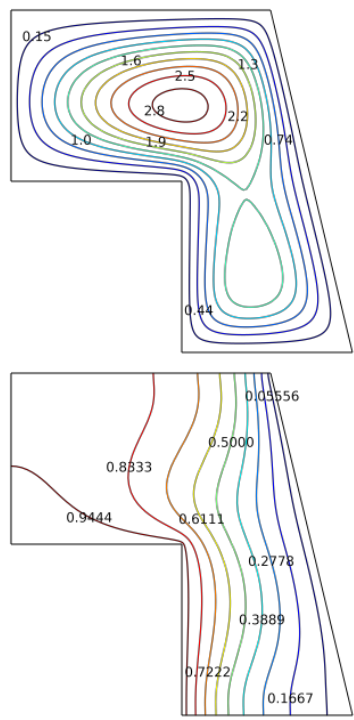

Fig3. Isotherm and streamlines for different Ha at $\mathrm{Ra}=105, \varphi=0.04$

As $R a=105$, the flow field shows a strong circulation, the appearance of roll rotating in the core region indicating the mode of free convection. But the flow is immobilized by a stable stratification doe to increasing of Hartmann numbers $(\mathrm{Ha}=100)$.

\subsection{Vertical velocity profile}

The vertical velocity profiles at $X=0.5$ are shown in figure 4 . The conical space region is surrounded by a convective roll rotating in a clockwise sense. Large velocity gradients indicating a boundary layer structure exist in regions adjacent to the thermally active (hot and cold) walls and isolated walls. When $\mathrm{Ha}>25$, the flow is week in the core of conical space., we observe an overall decrease in the velocities.

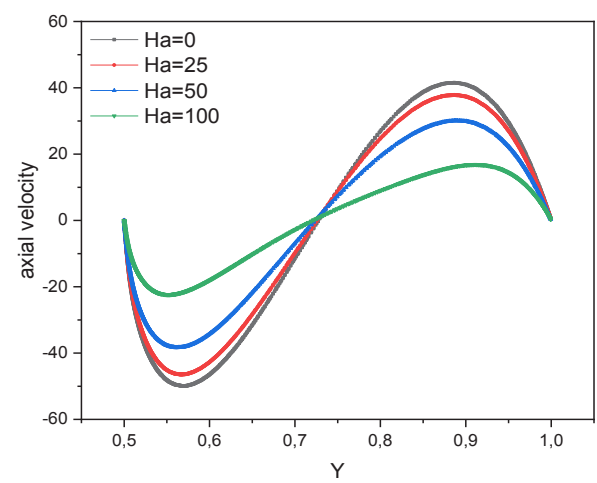

Fig. 4: Variation of vertical velocity component along middle horizontal line of the enclosure
Figure 5 shows the variation in average Nusselt number according to Rayleigh number $(\mathrm{Ra}=103,104,105$, and 106) at different values of Hartmann number $(\mathrm{Ha}=0,25$, 50 and 100) for solid volume fraction equal to 0.04 . We observe that average Nusselt number increase for various Hartmann number with increasing of Rayleigh numbers.

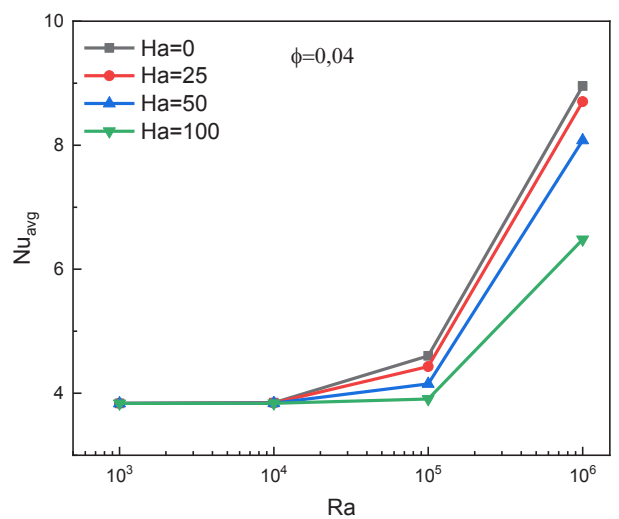

Fig05: Nusselt number for different Rayleigh number at $\varphi=0.04$

\section{Conclusion}

Natural convection in a conical cavity filled with a $\mathrm{Cu}-$ water nanofluid and under the action of an external magnetic field has been numerically studied.

The effect of magnetic field on flow and heat transfer rate was examined.

The main results are:

- The magnetic field reduces circulation in the conical cavity.

- When the conductive regime is dominant $\left(\mathrm{Ra}=10^{3}\right)$, the intensity of the magnetic field have a small effect on the heat transfer rate for all values of $\mathrm{Ha}$.

-The thermal performance increases with the augmentation of $\mathrm{Ra}$ and reduces with the enhance Ha.

\section{Reference;}

[1]. M. Behnia, J. A. Reizes and G. De Vahl Davis, Int. J. Numer. Methods Fluids, vol. 10, 1990.

[2]. C. Balaji and S. P. Venkateshan, Int. J. Heat and Fluid Flow, vol. 14, n 3, 1993.

[3]. H. Nouanegue, A. Muftuoglu and E. Bilgen, Int. J. Heat Mass Transfer, vol. 51, 2008.

[4]. C. Balaji, S.P. Venkateshan, Int. J. Heat Fluid Flow 14 (3) (1993).

[5]. K. Velusamy, T. Sundararajan and K. Seetharamu, Journal of Heat Transfer, vol. 123, 2001.

[6]. A. Mezrhab, H. Bouali, H. Amaoui, M. Bouzidi, Appl. Energy 83 (2006).

[7]. K. Khanafer, K. Vafai, M. Lightstone, Int. J. Heat Mass Transfer 46 (2003). 
[8]. Sheikha M. Al-Weheibi , M.M. Rahman , M.S. Alam , K. Vajravelu, International Journal of Mechanical Sciences (2017).

[9]. H.C. Brinkman, J. Chem. Phys. 20 (1952).

[10]. H. Sajjadi, A. Amiri Delouei, M. Sheikholeslami, M. Atashafrooz, S. Succi, Physica A: Statistical Mechanics and its Applications 515, 2019. 